\title{
Cerebral arterial angioplasty in a patient with Loeys-Dietz syndrome
}

\author{
Christopher P Kellner, ${ }^{1}$ Eric S Sussman, ${ }^{1}$ Christopher Donaldson, ${ }^{1}$ \\ E Sander Connolly Jr, ${ }^{2}$ Philip M Meyers ${ }^{1}$
}

${ }^{1}$ Department of Neurosurgery, Columbia University, New York, New York, USA ${ }^{2}$ Department of Neurological Surgery, Columbia University, New York, New York, USA

\section{Correspondence to} Dr Christopher P Kellner, christopher.kellner@gmail.com

Republished with permission from BMJ Case Reports Published 6 January 2014; doi:10.1136/bcr-2013-010857

Published Online First 15 January 2014

\section{CrossMark}

To cite: Kellner $C P$, Sussman ES, Donaldson C, et al. J Neurolntervent Surg 2015;7:e2.

\section{ABSTRACT}

A 14-year-old boy with Loeys-Dietz syndrome (LDS) had an acute neurologic decline 6 days after a subarachnoid hemorrhage. Cerebral angiography at presentation did not show an aneurysmal source of the hemorrhage. However, on post-bleed day 6 the patient experienced an acutely worsening headache and subsequently lost consciousness. Head CT showed new subarachnoid blood and repeat angiography demonstrated a basilar tip aneurysm. Endovascular coil embolization was performed and his neurologic status improved postoperatively until post-bleed day 9 when he became unresponsive. A CT angiogram demonstrated severe proximal vasospasm. After an unsuccessful attempt to treat the vasospasm medically, the patient was transported to the neurointerventional suite for intra-arterial vasodilator treatment, which also failed to ameliorate the vasospasm. The endovascular surgeons were then faced with the conundrum of attempting a high-risk cerebral angioplasty in a pediatric patient with LDS or returning to maximal medical treatment for severe refractory vasospasm.

\section{BACKGROUND}

Loeys-Dietz syndrome (LDS) is a rare connective tissue disorder (CTD) characterized by cardiovascular, craniofacial, skeletal and neurocognitive abnormalities. ${ }^{1}$ Patients with LDS also have diffuse vascular abnormalities including an increased incidence of intracranial aneurysms. ${ }^{2}{ }^{3}$ Due to the inherent vascular fragility in any $\mathrm{CTD}^{4-6}$ along with the significant vascular tortuosity characteristic of LDS, intracranial aneurysms represent a unique challenge in this patient population. There are a limited number of case reports in the literature describing successful treatment of unruptured LDS-associated aneurysms using both open microsurgical clipping ${ }^{3}$ and endovascular coiling. ${ }^{7}$ This is the first report of a successfully treated ruptured aneurysm, as well as the first report of successful angioplasty for refractory vasospasm in a patient with LDS.

\section{CASE PRESENTATION}

A 14-year-old boy with LDS presented with a 1-day history of headache accompanied by nausea/ vomiting. The location of the headache was occipital without radiation, and the pain was increasing in severity. He denied associated fever, diarrhea, abdominal pain or focal neurologic symptoms. $\mathrm{He}$ also denied any neck trauma, including hyperextension or rotation.
His past medical history included myxomatous mitral/tricuspid valves with aortic root dilation. His past surgical history included clubfoot repair at 1 year. All immunizations and vaccinations were up to date and there were no known allergies.

Physical examination showed an otherwise healthy young man with Marfanoid features who was afebrile with stable vital signs and a Glasgow Coma Scale (GCS) score of 14. The patient was intact and neurologic examination was non-focal; however, consciousness declined in the emergency room, necessitating intubation.

Emergency non-contrast head CT (NCHCT) showed diffuse subarachnoid hemorrhage (modified Fisher grade 3; figure 1A). Moderately severe hydrocephalus was also noted (figure 1B). Diagnostic cerebral angiography performed after placement of a right frontal external ventricular drain did not indicate an underlying aneurysm (figure 2).

The patient was transferred to the neurointensive care unit (NICU) where his neurologic status steadily improved. On post-bleed day (PBD) 6 the patient experienced acute-onset severe headache and altered mental status that rapidly progressed to loss of consciousness. NCHCT showed acute rebleeding, with increased blood in the basal cisterns bilaterally and in the third and fourth ventricles. A left frontal external ventricular drain was placed and the patient was transported to the neurointerventional suite. Repeat angiography showed a $4 \times 4 \times 3 \mathrm{~mm}$ basilar apex aneurysm projecting ventrally (figure $3 \mathrm{~A}$ ), which was treated by endovascular coil embolization. Subsequent arteriography showed complete occlusion of the aneurysm (figure 3B).

The patient was transferred back to the NICU in a stable condition. He was able to follow commands until PBD 9 when he was found to be aphasic, stuporous and unable to follow commands. The patient was sent for further radiographic investigation to assess for possible hydrocephalus, vasospasm, rebleed or stroke.

\section{INVESTIGATIONS}

NCHCT demonstrated no significant changes compared with the previous scan. CT angiography (CTA) showed moderate to severe narrowing of the right supraclinoid internal carotid artery and narrowing of the proximal anterior and middle cerebral arteries bilaterally. Catheter angiography showed severe bilateral large vessel symptomatic vasospasm (figure 4A). 


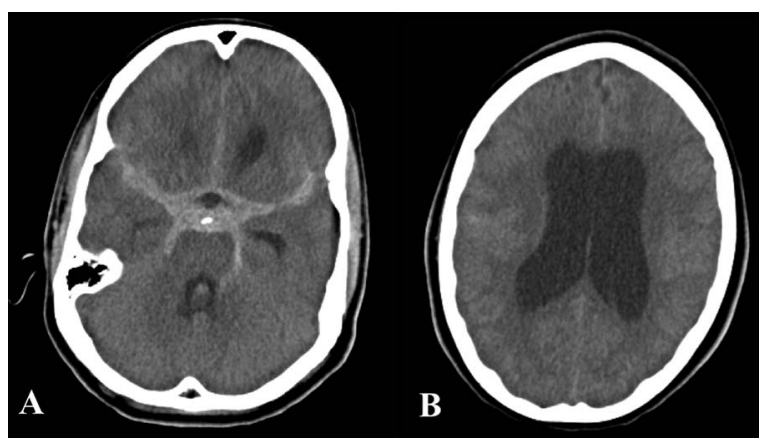

Figure 1 (A) Non-contrast head CT scan obtained at the time of admission, demonstrating substantial subarachnoid hemorrhage involving the basal, suprasellar and sylvian cisterns and the interhemispheric fissure. (B) A higher slice in the admission head CT scan demonstrating significant dilation of the bilateral lateral ventricles.

\section{DIFFERENTIAL DIAGNOSIS}

The differential diagnosis at the time of the delayed neurologic decline (PBD 9) was: (1) hydrocephalus; (2) vasospasm; (3) rebleed; (4) thromboembolic stroke; (5) seizure; or (6) infection.

\section{TREATMENT}

The primary concerns were hydrocephalus and vasospasm. NCHCT did not show ventricular dilation. CTA demonstrated diffuse vasospasm of the large cerebral arteries. A trial of increased blood pressure was attempted with no improvement. Intra-arterial verapamil also failed to ameliorate the vasospasm (figure 4A). Thus, balloon angioplasty of the vasospastic vessels was performed.

This procedure was carried out using a standard balloon angioplasty technique. An EV3 HyperGlide $3 \times 10 \mathrm{~mm}$ angioplasty balloon was prepared with a 0.010 inch X-pedion microguidewire to guide the catheter sequentially into each of the target vessels. Following gentle balloon angioplasty, intra-arterial verapamil (1 mg/mL normal saline) was infused to augment the mechanical treatment.

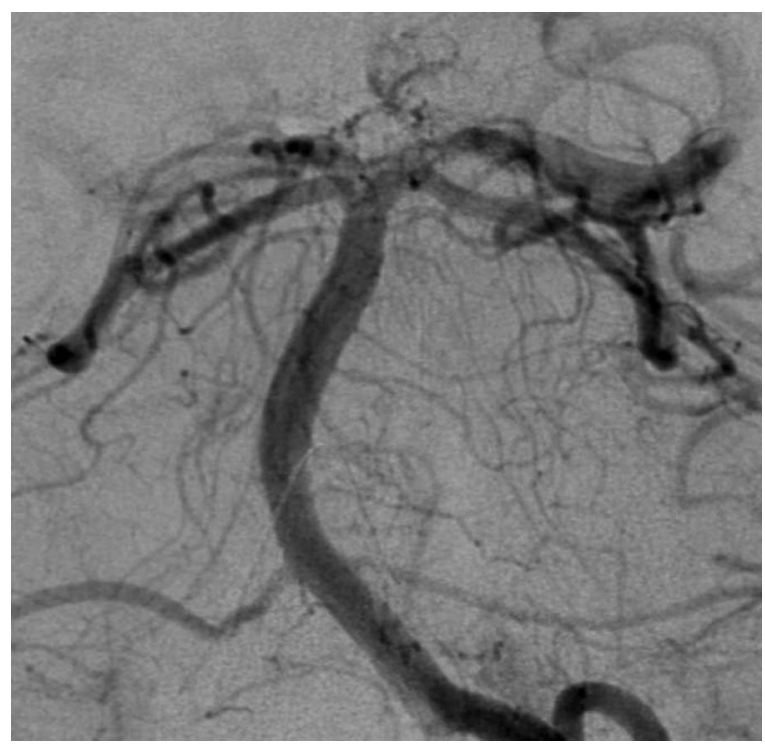

Figure 2 Cerebral angiography with a right vertebral artery injection demonstrating posterior circulation of normal appearance with no visible aneurysms.

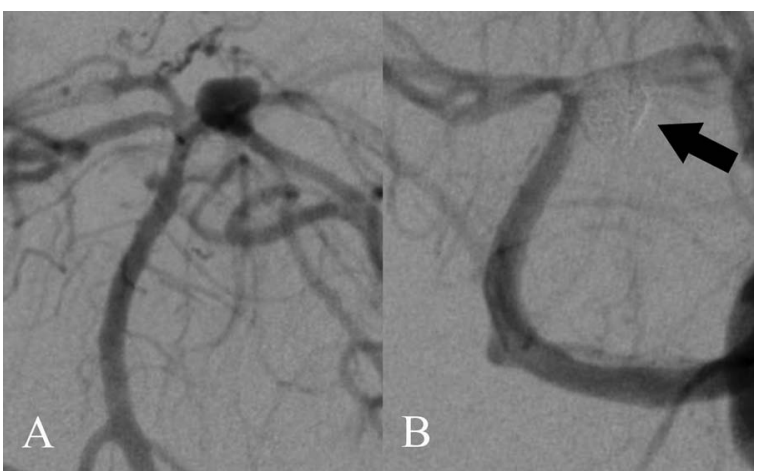

Figure 3 (A) Cerebral angiography with a left vertebral artery injection illustrating a ventrally projecting basilar artery apex aneurysm that was not visible on the angiogram 6 days previously. (B) Angiographic imaging obtained immediately after coil embolization of basilar artery apex aneurysm, demonstrating complete aneurysmal occlusion.

Despite concerns that the patient's CTD would predispose to vessel injury during balloon angioplasty, the procedure was well tolerated without any unintended iatrogenic cerebral branch occlusion, contrast extravasation or new mass effect. Post-procedural imaging demonstrated marked improvement of radiographic vasospasm (figure $4 \mathrm{~B}$ ).

\section{OUTCOME AND FOLLOW-UP}

Follow-up CTA on PBDs 10, 12 and 13 showed mild residual vasospasm and durable occlusion of the basilar tip aneurysm. There have been no additional neurointerventional procedures.

\section{DISCUSSION}

Many CTDs, including LDS, carry an increased risk of cerebral aneurysm formation, ${ }^{3}$ and retrospective data indicate that LDS-associated aneurysms have a higher risk of rupture than equivalent-sized aneurysms in the general population. ${ }^{6}$ Consequently, there has been a trend towards early and aggressive surgical intervention for known unruptured LDS-associated aneurysms. Although the optimal surgical management of

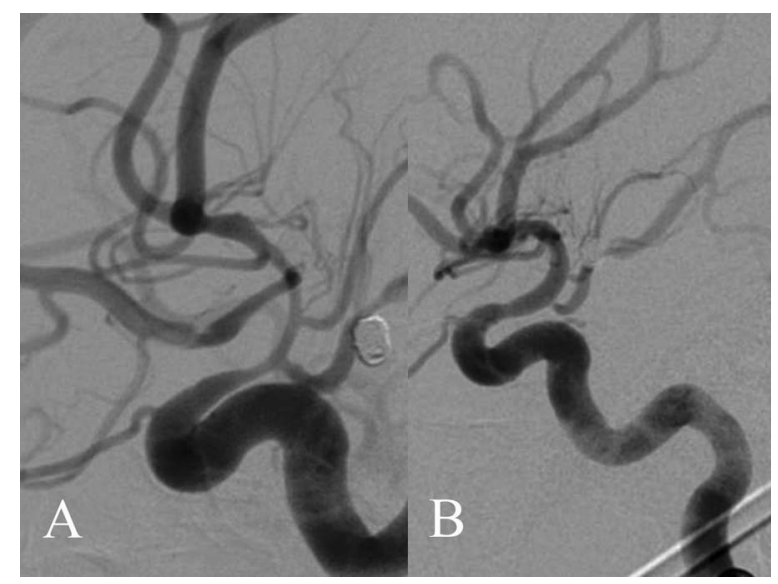

Figure 4 (A) Cerebral angiography with a left internal cerebral artery injection illustrating widespread vasospasm in the anterior cerebral circulation, most pronounced in the supraclinoid internal carotid artery (ICA). (B) Angiographic image obtained immediately after angioplasty and vasodilator infusion therapy showing marked improvement of supraclinoid ICA vasospasm. 
LDS-associated aneurysms is not well-established, relevant case reports emphasize the advantages of an open microsurgical approach. ${ }^{3}$ The justification is that CTDs are associated with a greater degree of vessel friability and fragility and therefore this patient population may be at increased risk of arterial injury with endovascular manipulation. In fact, there have been a number of case reports of significant morbidity/mortality following endovascular interventions in patients with CTDs. ${ }^{8} 9$ Nonetheless, Levitt et $a l^{7}$ recently demonstrated success in coiling such aneurysms in two patients with LDS, indicating that an endovascular approach may be considered in carefully selected LDS patients.

Patients with LDS presenting with ruptured intracranial aneurysms comprise a distinct patient population in whom endovascular intervention may be considered. The literature strongly supports early surgical intervention in aneurysmal subarachnoid hemorrhage to reduce the risk of hemorrhage ${ }^{10}$; thus, in patients with aneurysmal anatomy that is particularly favorable for an endovascular approach, the benefit of a familiar approach may outweigh the risks associated with endovascular manipulation. Furthermore, in patients with severe refractory

\section{Key messages}

- The differential diagnosis for acute neurologic change following subarachnoid haemorrhage is broad and includes rebleeding, vasospasm, hydrocephalus, thromboembolic stroke, seizure and infection.

- Cerebral vasospasm is an active process of vasoconstriction with serious neurologic consequences that must be treated quickly with rapid escalation to endovascular intervention if necessary.

- Cerebral angioplasty is a high-risk but feasible procedure for the management of vasospasm in patients with Loeys-Dietz syndrome and should be cautiously considered when all other treatment options have been exhausted. vasospasm, more aggressive endovascular intervention (ie, angioplasty) may be indicated.

In summary, our experience supports the previous report by Levitt et $\mathrm{al}^{7}$ which demonstrated the safety and feasibility of neuroendovascular intervention in carefully selected patients with LDS. Furthermore, this is the first report of cerebral angioplasty being performed in a patient with LDS, suggesting that this is a viable treatment option in patients with LDS with severe refractory vasospasm.

Contributors PMM was the primary attending involved in the care of the patient whose case was presented. He revised the paper and is the guarantor. CPK was the primary resident involved in the care of the presented patient. He also was the primary writer of the paper. ESS and CD contributed background research, assistance with drafting of the paper and revisions of the paper.

Competing interests None.

Patient consent Obtained.

Provenance and peer review Not commissioned; externally peer reviewed

\section{REFERENCES}

1 Loeys BL, Chen J, Neptune ER, et al. A syndrome of altered cardiovascular, craniofacial, neurocognitive and skeletal development caused by mutations in TGFBR1 or TGFBR2. Nat Genet 2005;37:275-81.

2 Rodrigues VJ, Elsayed S, Loeys BL, et al. Neuroradiologic manifestations of Loeys-Dietz syndrome type 1. AJNR Am J Neuroradiol 2009;30:1614-19.

3 Rahme RJ, Adel JG, Bendok BR, et al. Association of intracranial aneurysm and Loeys-Dietz syndrome: case illustration, management, and literature review. Neurosurgery 2011;69:488-93.

4 Loeys BL, Schwarze U, Holm T, et al. Aneurysm syndromes caused by mutations in the TGF-beta receptor. N Engl J Med 2006;355:788-98.

5 Aalberts JJJ, van den Berg MP, Bergman JEH, et al. The many faces of aggressive aortic pathology: Loeys-Dietz syndrome. Neth Heart J 2008;16:299-304.

6 Hughes BD, Powers CJ, Zomorodi AR. Clipping of a cerebral aneurysm in a patient with Loeys-Dietz syndrome: case report. Neurosurgery 2011;69:E746-55.

7 Levitt MR, Morton RP, Mai JC, et al. Endovascular treatment of intracranial aneurysms in Loeys-Dietz syndrome. J Neurointerv Surg 2012;4:e37.

8 Horowitz MB, Purdy PD, Valentine RJ, et al. Remote vascular catastrophes after neurovascular interventional therapy for type 4 Ehlers-Danlos syndrome. AJNR Am J Neuroradiol 2000;21:974-6.

9 Usinskiene J, Mazighi M, Bisdorff A, et al. Fatal peritoneal bleeding following embolization of a carotid-cavernous fistula in Ehlers-Danlos syndrome type IV. Cardiovasc Intervent Radiol 2006;29:1104-6.

10 Peerless SJ, Hernesniemi JA, Gutman FB, et al. Early surgery for ruptured vertebrobasilar aneurysms. J Neurosurg 1994;80:643-9. 